\title{
Diet-Induced Obesity: When Does Consumption Become Overconsumption?
}

\author{
Stephanie H. Fay • Graham S. Finlayson • Neil A. King
}

Published online: 11 October 2012

(C) Springer Science+Business Media New York 2012

\begin{abstract}
Overconsumption is commonly implicated in the etiology of obesity; however there is a lack of consensus on a definition and the most appropriate methodology for assessing it. The aim of this communication is to highlight the need for theoretical consensus on the assessment of overconsumption, which may lead to improved methodological standards in obesity research. In laboratory studies, overconsumption is most frequently inferred from the comparison of food intake within or between individuals against a single control. Measurement often relies on a single eating episode with limited consideration of preceding or subsequent intake. An alternative approach is to consider food intake in the context of energy requirements, within an energy balance framework. One such marker of chronic overconsumption is body weight. There is a need for agreement on the definition and measurement of overconsumption, so that its role in weight gain and obesity can be more precisely delineated.
\end{abstract}

Keywords Overconsumption · Obesity · Methodological assessment $\cdot$ Hyperphagia $\cdot$ Diet $\cdot$ Consumption

\section{Introduction: Relevance of Overconsumption to Obesity}

Overconsumption of food is a widely discussed phenomenon with reference to the etiology of weight gain and

\section{S. H. Fay $(\bowtie) \cdot$ N. A. King}

Institute of Health and Biomedical Innovation, Queensland University of Technology,

60 Musk Avenue,

Brisbane, Queensland 4059, Australia

e-mail: Stephanie.fay@qut.edu.au

\section{G. S. Finlayson}

Institute of Psychological Sciences,

University of Leeds,

Leeds, UK obesity. A positive energy balance driven by dietary overconsumption is thought to largely account for the marked increase in the prevalence of obesity [1-3]. However, for a term so widely used in obesity research, the concept of overconsumption remains surprisingly ill-defined. It is by definition a relative term; but relative to what? At what point does 'consumption' become 'overconsumption', and when is it significant and meaningful with respect to weight gain and obesity?

Understanding the etiology of obesity is vital for effective treatment and prevention. It is proposed that research examining factors in the etiology of obesity, such as overconsumption, must do so within an energy balance framework [4•]. It is agreed that when energy intake consistently exceeds expenditure a positive energy balance and weight gain will occur; increased energy intake that is matched by increased energy expenditure, or a compensatory reduction in intake, will not. Therefore, research into overconsumption must necessarily consider its relationship with energy requirements, even if it is posited that energy intake is the most potent driver of imbalance.

\section{Current Assessment of Overconsumption}

Currently, no formal scientific definition of overconsumption exists, and the methodologies used in studies that claim to measure overconsumption differ substantially. Therefore, comparison between studies is difficult, and the role of overconsumption in the etiology of obesity is obscured.

Overconsumption is commonly studied in experimental paradigms by comparing food intake between two independent groups of participants. In this way, the lower energy intake is used as benchmark, against which significantly higher energy intake is often classed as 'overconsumption'. While a complete review of the literature is beyond the scope of this paper, several laboratory studies have used 
comparisons in this way to assess overconsumption. For example, ad libitum food intake has been compared between participants identified as being high and low in sensitivity to food reward (e.g., [5]), high and low in dietary restraint (e.g., [6]), disinhibition (e.g., [7]) or self control (e.g., [8]). A related paradigm compares intake between-subjects or within-subjects in a control condition with those subjected to an experimental manipulation, in order to determine its effects upon propensity to overconsume (e.g., [9-11]).

While illustrative, these comparative approaches to assessing overconsumption pose several problems. Although the laboratory environment allows high precision, unfamiliar conditions and possibly foods may provoke atypical eating behaviors [12], so that observation of a single episode of eating behavior in the laboratory is not a guarantee that it is typical of the individual. More importantly, high levels of individual differences in compensatory behaviors, habitual diet and activity-induced energy expenditure are likely to negate any sustained differences in caloric intake observed in a laboratory. It can be argued that it is meaningless to compare absolute food consumption between subjects, as energy intake and expenditure are so highly variable between individuals. Therefore, the preferred method of assessing overconsumption should be within the context of each individual, with reference to individual energy requirements. However, the issue of for how long consumption should be tracked in order to reveal meaningful changes remains to be addressed.

\section{What is an Appropriate Time Period for Determining Overconsumption?}

Evidence has demonstrated that food consumption is highly temporally variable within, as well as between, individuals $[13,14]$. A major issue in the prevailing measurement of overconsumption is that assessment of energy intake is often limited to a single eating episode, but the reliability of these measures is rarely assessed (although see [15]). Examining food consumption on an 'episode by episode' basis may mirror how obesity can occur ('It's only one small piece of cake;' 'It's only one extra glass of wine,' etc.), if each episode is sufficiently repeated. However, in terms of overall risk for obesity, it is clear that the frequency and magnitude of overconsumption episodes are fundamentally important.

Moreover, an immediate limitation of relying on a single assessment is that there is often little or no consideration of the individual's eating and activity behaviors outside the laboratory. When considering risk factors for overweight and obesity, the key issue is therefore whether episodes of overconsumption are compensated for by subsequent behavioral adjustments. Therefore, overconsumption as defined by a single eating episode is insufficient and invalid to classify a person as an 'overconsumer'. That is, a person may be at greater risk of weight gain, but it is not inevitable if the individual is able to regulate their eating or activity to compensate for episodic overconsumption. Only a small number of studies to date have examined behavioral compensation for laboratory food intake (e.g., [16, 17]).

Overall energy balance, including compensation for changes in energy intake or expenditure, can be observed only over a longer period [18]. Therefore, when drawing conclusions of practical and clinical significance, instances of overconsumption relative to energy requirements must be examined on multiple occasions over a longer time period.

\section{Body Weight and Composition as Markers of Overconsumption}

In the context of chronic energy imbalance, a robust and objective marker of overconsumption is an increase in body weight. It is acknowledged that in the short term, body weight is influenced by hydration levels, and that weight changes conceal changes in body composition. Increased body weight implies a chronic positive energy balance, which results from an excess of energy consumed relative to need (overconsumption). However, simply tracking body weight or fat mass will conceal the acute episodic processes and mechanisms that cause overconsumption. Further, body weight and composition are unlikely to alter measurably in response to single overconsumption episodes. Therefore, while body weight is a useful indicator of overconsumption, it should be applied with caution.

\section{Overconsumption Assessed Relative to Energy Requirements}

As discussed, research is increasingly concluding that overconsumption and obesity must be considered within an energy balance framework [4•]. Only consumption that consistently exceeds energy requirements will lead to a positive energy balance, and for analysis of its role in the etiology of weight gain, it is critical that food intake is considered in the context of individual energy requirements.

Currently, this approach is applied in very few contexts. Overfeeding studies tend to consider total energy requirements when determining how much surplus energy to add to the habitual diet (e.g., [19, 20]). Similarly, food consumed within a single eating episode may be considered as a proportion of total daily intake. This approach is utilized by food manufacturers to indicate recommended serving sizes, which are based on a nominal percentage of the average adult's daily energy intake requirements. While this 
rough estimate may serve as a consumer guide, it is insufficient and invalid for the purposes of research into overconsumption; not least as it is based on a single-episode assessment and ignores issues of individual variability. Further, the percentage required for consumption in a single session to be excessive (relative to energy requirement) is necessarily subject to a range of factors. For example, if the food eaten comprises the main meal, it would be expected to contain a larger proportion of the day's energy, whereas the same percentage of total energy intake extra-meal (i.e., as a snack) might indicate an excess. These issues highlight the problem of assessing overconsumption within a single eating episode, and imposing a daily time-frame on total energy requirements.

\section{Conclusions}

In summary, while overconsumption is a relative term, it is only meaningful for obesity risk when considered relative to individual energy requirement. Only food intake that consistently exceeds energy expenditure will foster a positive energy balance and lead to weight gain. At present, surprisingly few laboratory studies of behavioral obesity research take these considerations into account in their design and interpretation. A standardized methodological platform to measure overconsumption is required. This would have important implications for clinical assessments. While it is somewhat premature to recommend a clearly defined platform for detecting overconsumption in a clinical setting, until such a platform is developed, the authors suggest that an individual's energy expenditure is assessed as a standard against which measured food intake, over a minimum of one week and on multiple occasions, can be compared as a relative percentage.

The issues raised here, together with the importance of the study of overconsumption in obesity research, suggest a need for further review and consensus on methodology. An accurate and testable working definition of overconsumption is necessary in order to properly investigate its role in the etiology of obesity. Further research within the field should give adequate consideration to the reliability and reproducibility of measures of food intake, as well as the validity of any measure within the wider context of its role in energy balance and weight gain.

Acknowledgments This manuscript was written as part of a $\mathrm{PhD}$ thesis supported by a Postgraduate Research Award, although did not receive explicit funding itself.

Disclosure No potential conflicts of interest relevant to this article were reported.

\section{References}

Papers of particular interest, published recently, have been highlighted as:

- Of importance

1. Duffey KJ, Popkin BM. Energy density, portion size, and eating occasions: contributions to increased energy intake in the United States, 1977-2006. PLoS Med. 2011;8(6):e10011050.

2. Levitsky DA, Pacanowski CR. Free will and the obesity epidemic. Public Health Nutr. 2011;15(5):126-41.

3. Swinburn BA, et al. Estimating the changes in energy flux that characterize the rise in obesity prevalence. Am J Clin Nutr. 2009;89(6):1723-8.

4. - Hall KD, et al. Energy balance and its components: implications for body weight regulation. Am J Clin Nutr. 2012;95(4):989-94. This article presents a compelling argument for consideration of overall energy balance.

5. Epstein LH, et al. Food reinforcement, the dopamine D-sub-2 receptor genotype, and energy intake in obese and nonobese humans. Behav Neurosci. 2007;121(5):877-86.

6. Provencher V, Polivy J, Herman CP. Perceived healthiness of food. If it's healthy, you can eat more! Appetite. 2009;52(2):340-4.

7. Westenhoefer J, et al. Cognitive control of eating behavior and the disinhibition effect. Appetite. 1994;23(1):27-41.

8. Guerrieri R, Nederkoorn C, Jansen A. The interaction between impulsivity and a varied food environment: its influence on food intake and overweight. Int J Obes. 2007;32(4):708-14.

9. Coelho JS, et al. Eating behavior in response to food-cue exposure: examining the cue-reactivity and counteractive-control models. Psychol Addict Behav. 2009;23(1):131-9.

10. Fay SH, Finlayson G. Negative affect-induced food intake in nondieting women is reward driven and associated with restraineddisinhibited eating subtype. Appetite. 2011;56(3):682-8.

11. McFarlane T, Polivy J, Herman CP. Effects of false weight feedback on mood, self-evaluation, and food intake in restrained and unrestrained eaters. J Abnorm Psychol. 1998;107(2):312-8.

12. Hill AJ, Rogers PJ, Blundell JE. Techniques for the experimental measurement of human eating behaviour and food intake: a practical guide. Int J Obes Relat Metab Disord. 1995;19(6):361-75.

13. de Castro JM. Weekly rhythms of spontaneous nutrient intake and meal pattern of humans. Physiol Behav. 1991;50(4):729-38.

14. Ma Y, et al. Number of 24 -Hour diet recalls needed to estimate energy intake. Ann Epidemiol. 2009;19(8):553-9.

15. Keene JM, Hope T. Hyperphagia in dementia: 1. The use of an objective and reliable method for measuring hyperphagia in people with dementia. Appetite. 1997;28(2):151-65.

16. Chaput J-P, Tremblay A. Acute effects of knowledge-based work on feeding behavior and energy intake. Physiol Behav. 2007;90 (1):66-72.

17. Wardle J, Beales S. Restraint and food intake: an experimental study of eating patterns in the laboratory and in normal life. Behav Res Ther. 1987;25(3):179-85.

18. Stubbs RJ, et al. The effect of graded levels of exercise on energy intake and balance in free-living women. Int J Obes Relat Metab Disord. 2002;26(6):866-9.

19. Bouchard C, et al. The response to long-term overfeeding in identical twins. N Engl J Med. 1990;322(21):1477-82.

20. Levine JA, Eberhardt NL, Jensen MD. Role of nonexercise activity thermogenesis in resistance to fat gain in humans. Science. 1999;283(5399):212-4. 